\title{
Leaky modes of a left-handed slab
}

\author{
A. Moreau \\ antoine.moreau@univ-bpclermont.fr \\ D. Felbacq
}

\begin{abstract}
LASMEA, UMR CNRS 6602, Université Blaise Pascal, 24 avenue des Landais, 63177 Aubière, France
GES, UMR CNRS 5650, Université de Montpellier II, Bat. 21, CC074, Place E. Bataillon, 34095 Montpellier Cedex 05, France.
\end{abstract}

Using complex plane analysis we show that left-handed slab may support either leaky slab waves, which are backward because of negative refraction, or leaky surface waves, which are backward or forward depending on the propagation direction of the surface wave itself. Moreover, there is a general connection between the reflection coefficient of the left-handed slab and the one of the corresponding right-handed slab (with opposite permittivity and permeability) so that leaky slab modes are excited for the same angle of incidence of the impinging beam for both structures. Many negative giant lateral shifts can be explained by the excitation of these leaky modes. [DOI: $10.2971 /$ jeos.2008.08032]

Keywords: left-handed materials, leaky modes, complex plane analysis

\section{INTRODUCTION}

Left-handed materials [1] have long been considered a theoretical oddity. Since it has been demonstrated that they could be produced using metamaterials [2], they have attracted much attention. The basic physics of left-handed materials (LHM) is truly exotic and has been completely ignored until recently, it renews the physics of lamellar structures to the extend that a bare slab of LHM exhibits many surprising properties : it can for instance support unusual guided modes [3, 4] or behave as a perfect lens [5]. In this paper, we study the exotic properties of the different types of leaky waves supported by a left-handed slab. Given the importance of the left-handed slab for both fundamental and applied works, there is obviously a need for a clear understanding of these properties.

We particularly show that two types of leaky waves are supported by such a structure (i) leaky slab waves which are always backward due to negative refraction and (ii) leaky surface waves which do not exist for a right-handed slab and which can be backward or forward. The excitation of these modes leads to positive or negative giant lateral shifts, the latter being rather exotic [6].

\section{LEAKY MODES AND GIANT LATERAL SHIFTS}

A leaky mode [6] is a solution of the wave equation which satisfies the dispersion relation of a structure but with a propagative solution above and (or) under the structure. Whereas a guided mode has a real propagation constant, the propagation constant of a leaky mode is complex because the energy of the waves leaks out of the structure and the waves is attenuated. A leaky wave is thus a complex solution of the dispersion relation and a complex plane analysis is thus particularly rele- vant for a thorough analysis of its properties. Let us underline that a leaky mode may be either forward, which is common, or backward, leading to a propagation constant which has a positive (respectively negative) imaginary part.

Let us consider a slab characterized by $\varepsilon_{2}$ and $\mu_{2}$ surrounded by right-handed media with $\varepsilon_{1}$ and $\mu_{1}$ (resp. $\varepsilon_{3}$ and $\mu_{3}$ ) above (resp. under) the slab as shown in Figure 1. The values we have chosen for $\varepsilon_{2}$ and $\mu_{2}$ are arbitrary but realistic [7] so that this structure could be realized using split-ring resonnators and wires.

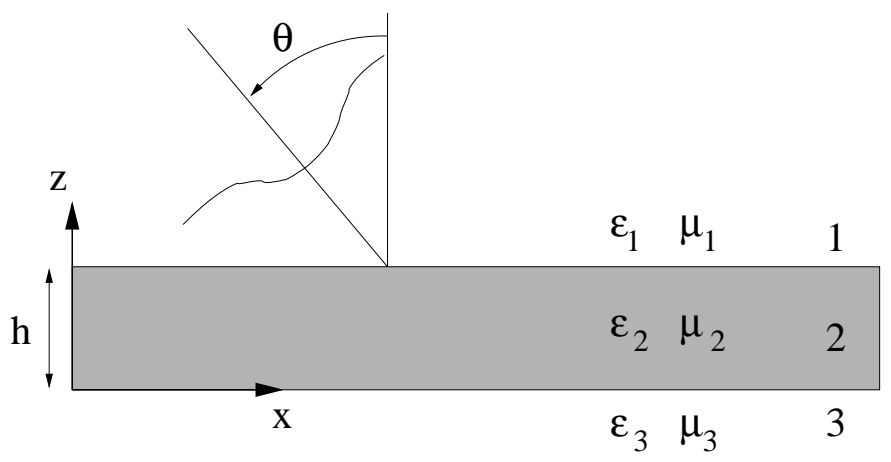

FIG. 1 The LHM slab of thickness $h$ surrounded by right-handed media.

We may assume that $\varepsilon_{1} \mu_{1} \geq \varepsilon_{3} \mu_{3}$ with no loss of generality.

The relation dispersion of such a structure can be written

$$
r_{21} r_{23}=\exp \left(-2 i \gamma_{2} h\right)
$$

where $\gamma_{i}=\sqrt{\varepsilon_{i} \mu_{i} k_{0}^{2}-\alpha^{2}}, k_{0}=\frac{\omega}{c}=\frac{2 \pi}{\lambda}$ and $r_{i j}=\frac{\kappa_{i}-\kappa_{j}}{\kappa_{i}+\kappa_{j}}$ with $\kappa_{i}=\frac{\gamma_{i}}{\mu_{i}}$ in TE polarization (or $\kappa_{i}=\frac{\gamma_{i}}{\varepsilon_{i}}$ in TM polarization). Since $\varepsilon_{1} \mu_{1} \geq \varepsilon_{3} \mu_{3}$ and we are concerned with leaky waves, 
we will only consider values of $\alpha$ such that $\alpha<\sqrt{\varepsilon_{1} \mu_{1}} k_{0}$, which means that the solution will always be propagative at least in medium 1.

Let us now consider the reflection coefficient of a plane wave $\exp (i(\alpha x+\gamma z-\omega t))$ coming from upwards with an angle of incidence $\theta$ so that $\alpha=n k_{0} \sin \theta$. Its reflection coefficient can be written

$$
r=\frac{r_{23} \exp \left(2 i \gamma_{2} h\right)-r_{21}}{1-r_{21} r_{23} \exp \left(2 i \gamma_{2} h\right)}
$$

using the above definitions.

It is obvious that when the dispersion relation is satisfied, then the reflection coefficient presents a pole. A leaky mode thus corresponds to a pole of the reflection coefficient. A zero, located on the other side of the real axis, corresponds to each pole. As we will see in the following, a zone where the phase of $r$ quickly varies lies between a pole and its corresponding zero. This zone crosses the real axis, so that the presence of a pole is responsible for a swift variation of the phase on the real axis.

When considering the reflection of a gaussian beam on a structure whose reflection coefficient has a modulus equal to one (so that it can be written $r=e^{i \phi}$ ), the lateral displacement of the reflected beam's barycenter along the interface is given by the well known formula

$$
\delta=-\frac{\mathrm{d} \phi}{\mathrm{d} \alpha}
$$

This lateral displacement is the sign that a leaky wave has been excited by the incident beam. The reflected beam then has two components : the part which is reflected by the first interface of the structure (whose barycenter is not particularly displaced) and the leaky wave itself [6]. The reflected beam is heavily distorted by the leaky wave and presents an exponentially decreasing tail so that its barycenter is largely displaced : this is the so-called giant lateral shift.

This effect is sometimes called a giant Goos-Hänchen effect, but in this case the shift is due to the excitation of a leaky mode [6] and not, as in the real Goos-Hänchen effect [8, 9], to the total reflection.

\section{THE LEFT-HANDED SLAB}

With left-handed materials, though, negative lateral shifts seem to be much more common [10]-[14] than once expected [6]. Here we will consider the case of a left-handed slab (i.e. if $\varepsilon_{2}<0$ and $\mu_{2}<0$ ) and explain why the leaky modes supported by such a structure are usually backward. Our explanations will be supported by a complex plane analysis of the leaky modes.

Here Eq. (2) for the reflection coefficient remains perfectly valid. We will now distinguish two cases : the case when the solution is propagative in the left-handed medium and the case when the solution is evanescent in region 2 .

\subsection{Leaky slab modes}

When the field is propagative in the left-handed slab, large negative lateral shifts have been reported but not interpreted [13]. These shifts are due to the excitation of leaky slab modes or Perot-Fabry resonances of the slab at non normal incidence. Such leaky modes have already been studied for a righthanded slab [15] and they can be considered as constructive interferences of the multiple beams which are produced by reflections on the interfaces of the slab. In the case of a lefthanded slab, since the first beam undergoes a negative refraction as shown in Figure 2 these constructive interferences will logically generate a backward leaky mode. We may thus conclude that the existence of such a backward leaky mode is linked to negative refraction.

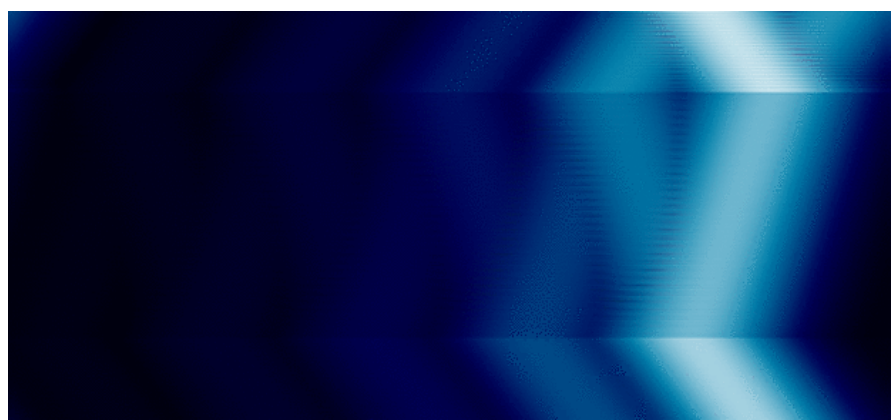

FIC. 2 Modulus of the field for a thick left-handed slab with $\epsilon_{1}=\epsilon_{3}=\mu_{1}=\mu_{3}=1$, $\epsilon_{2}=-3, \mu_{2}=-1$ and $h=60 \lambda$ using a gaussian incident beam with a waist of $20 \lambda$ and an incidence angle of $\theta=45^{\circ}$.

This argument is not a proof though : unexpected lateral shifts have been reported when the beams interfere destructively [16]. But if the leaky modes are backward, then the corresponding solutions of the dispersion relation and the poles of the reflection coefficient should have a negative imaginary part. This is what is shown in Figure 3.

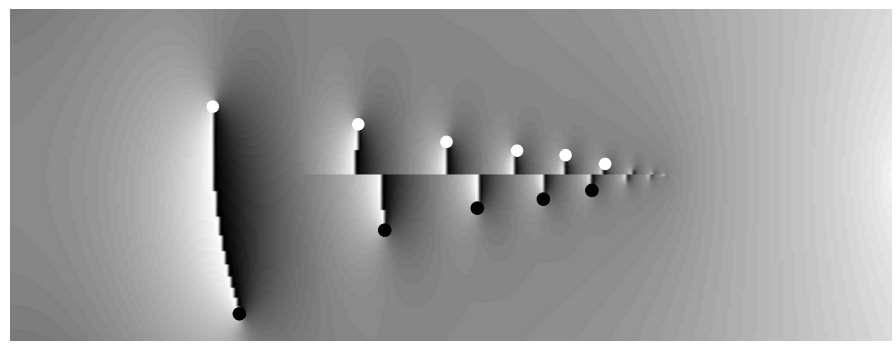

FIG. 3 The phase of the reflection coefficient in a part of the complex plan $\left[0, n_{1} k_{0}\right]+$ $i\left[-\frac{k_{0}}{\pi}, \frac{k_{0}}{\pi}\right]$. Each black point represents a pole and each white point a zero. The cut line is clearly visible here. The rapid variation of the phase which is due to each pole is obvious.

Two types of leaky slab waves should be distinguished (i) $L_{2}$ waves which are leaky in both the upper and the lower media and (ii) $L_{1}$ waves which are leaky only in the upper medium and evanescent in the lower one. The latter correspond to the poles located under the cut line.

Using complex plane analysis we will now try to show that all the solutions of the dispersion relation (1) are located in the lower part of the complex plane, meaning that all the leaky modes are backward. 
When the dispersion relation is satisfied, then the following condition holds :

$$
\left|r_{23} r_{21}\right|=e^{2 \gamma_{2}^{\prime \prime} h}
$$

As demonstrated in the annex, $\left|r_{i j}\right|>1$ whenever one of the media is left-handed. Since medium 2 is left-handed then the condition

$$
e^{2 \gamma_{2}^{\prime \prime} h}>1
$$

should be satisfied, which is possible for $\gamma_{2}^{\prime \prime}>0$ and therefore for $\alpha^{\prime \prime}<0$ (see the annex for details). The fact that $r_{i j}>1$ is thus the main reason why the poles of $r$ are under the axis and why the leaky slab modes are backward.

We must underline the fact that our demonstration is valid only for the first Riemann sheet : our proof cannot exclude that there may be some poles on the other Riemann sheet above the real axis, corresponding to forward $L_{1}$ leaky slab waves when $\varepsilon_{1} \mu_{1}>\varepsilon_{3} \mu_{3}$, but we could not find any.

\subsection{Leaky surface modes}

Let us now consider the situation in which the field is evanescent in the left-handed medium. Then $\gamma_{2}$ is purely imaginary on the real axis. Since $e^{2} \gamma_{2}^{\prime \prime} h$ tends towards infinity when $h \rightarrow+\infty$ then Eq. (4) can be satisfied only if $r_{23}$ has a pole $\left(r_{21}\right.$ cannot have one since the field is always propagative in the upper medium). This means that the structure may support a leaky mode only if the interface between medium 2 and 3 can support a guided mode. It is now well-known that such an interface actually supports a surface mode $[17,18]$ which can, depending on media 2 and 3, be backward (resp. forward) corresponding to a pole under the real axis (resp. above the real axis but on the other Riemann sheet). The leaky wave always has the same propagation direction as the surface mode, whatever the thickness of the slab, as shown in Figure 4. In the case of a forward leaky wave, only the zero belongs to the first Riemann sheet, just under the real axis. The pole shown in Figure 4 belongs to the other Riemann sheet.

Figure 5 finally shows the excitation of a backward leaky sur-
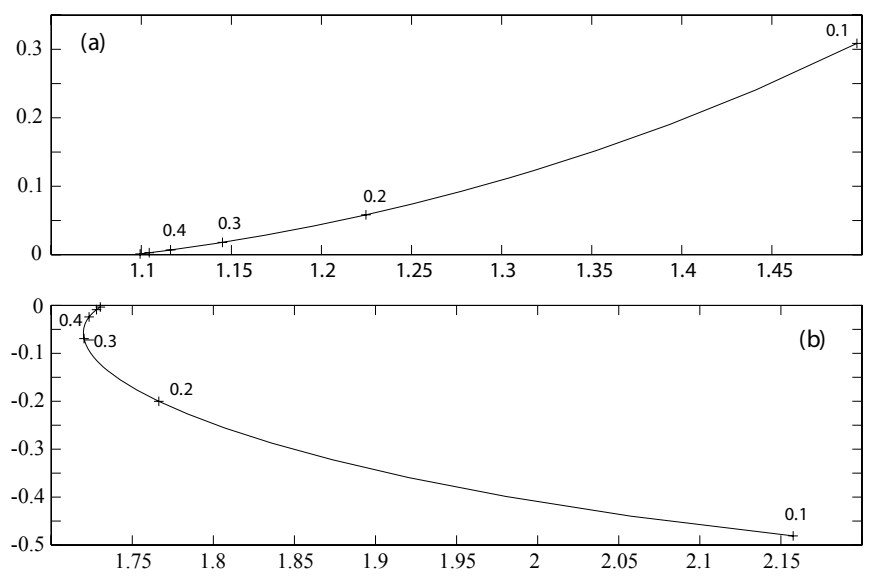

FIG. 4 Location of the poles in the $\frac{\alpha}{k_{0}}$ complex plane for different values of $h$ with $\varepsilon_{1}=9, \mu_{1}=\mu_{3}=\varepsilon_{3}=1$ and (a) $\varepsilon_{2}=-0.5$ and $\mu_{2}=-1.5$, showing a forward surface mode and (b) $\varepsilon_{2}=-5$ and $\mu_{2}=-0.5$, showing a backward surface mode.

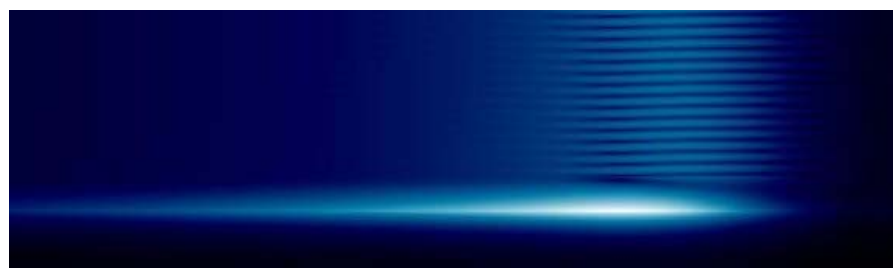

FIG. 5 Modulus of the field for a left-handed slab with $\epsilon_{1}=9, \epsilon_{3}=\mu_{1}=\mu_{3}=1$, $\epsilon_{2}=-0.5, \mu_{2}=-1.5$ and $h=0.6 \lambda$ using a gaussian incident beam with a waist of $20 \lambda$ and an incidence angle of $\theta=21.496^{\circ}$. The pole corresponding to the leaky mode is located at $\alpha_{p}=(1.0993+0.001267 i) k_{0}$.

face wave by a gaussian beam. The chosen values of $\mu_{2}$ may be obtained with simple split ring resonators [19] for instance.

\section{FUNDAMENTAL PROPERTY}

Let us a consider a structure with left-handed materials. We will call corresponding right-handed structure the structure obtained by replacing any left-handed medium by a medium with opposite permittivity and permeability, without changing the geometrical parameters.

In this section, we will concentrate on the link between the reflection coefficient of a left-handed slab and the one of its corresponding right-handed structure.

Let us consider the interface between a right-handed medium labelled $i$ and a left-handed medium $j$. The reflection coefficient of such an interface is $r_{i j}$. We will now define $r_{i j}^{+}$the reflection coefficient of an interface between medium $i$ and right-handed medium characterized by $\left|\varepsilon_{j}\right|$ and $\left|\mu_{j}\right|$. It is not difficult to see, from the expression of $r_{i j}$ that

$$
r_{i j}^{+}=\frac{1}{r_{i j}} .
$$

This allows us to understand why the Goos-Hänchen shift of an interface between a right- and a left-handed medium is the opposite of the corresponding right-handed structure [11] since the phases of both structures are opposite on the real axis.

The reflection coefficient $r$ can now be written

$$
\begin{aligned}
r & =\frac{\frac{e^{2 i \gamma_{2} h}}{r_{23}^{+}}-\frac{1}{r_{21}^{+}}}{1-\frac{e^{2 i \gamma_{2} h}}{r_{21}^{+} r_{23}^{+}}} \\
& =\frac{r_{23}^{+} e^{-2 i \gamma_{2} h}-r_{21}^{+}}{1-r_{21}^{+} r_{23}^{+} e^{-2 i \gamma_{2} h}}
\end{aligned}
$$

Since $\sqrt{z^{*}}=\sqrt{z}^{*}$ except when $z$ is on the cut line, then $\gamma\left(z^{*}\right)=\gamma(z)^{*}$ and hence $r_{i j}^{+}(z)^{*}=r_{i j}^{+}\left(z^{*}\right)$ so that

$$
r(z)^{*}=\frac{r_{23}^{+}\left(z^{*}\right) e^{2 i \gamma_{2}\left(z^{*}\right) h}-r_{21}^{+}\left(z^{*}\right)}{1-r_{21}^{+}\left(z^{*}\right) r_{23}^{+}\left(z^{*}\right) e^{2 i \gamma_{2}\left(z^{*}\right) h}},
$$

which can simply be written

$$
r(z)^{*}=r^{+}\left(z^{*}\right)
$$


where $r^{+}$is the coefficient reflection of the corresponding right-handed slab. Note that this relation does not hold on the cut line, but that it holds for the two Riemann sheets. This means that the poles of the left-handed slab and the poles of the corresponding right-handed slab are symmetrical with respect to the real axis. This means that $L_{2}$ waves can be excited for the same incidence angle for both structures. This is not the case for $L_{1}$ modes : the function $r$ on the real axis is continuous with the lower part of the first Riemann sheet whatever the situation and the poles which are above the cut line thus have no effect on the real axis.

As an example, we have computed the field in TE polarization inside and around the slab when it is illuminated with a gaussian beam for the left-handed slab and its corresponding right-handed structure. The results are shown in Figures 6 and 7.

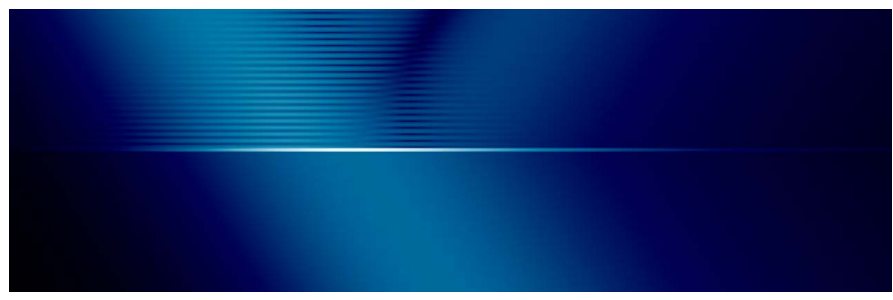

FIG. 6 Modulus of the field for a symmetrical slab with $\epsilon_{1}=\epsilon_{3}=9, \mu_{1}=\mu_{3}=1$, $\epsilon_{2}=1.5, \mu_{2}=1$ and $h=1.3 \lambda$ using a gaussian incident beam with a waist of $20 \lambda$ and an incidence angle of $\theta=22.78^{\circ}$.

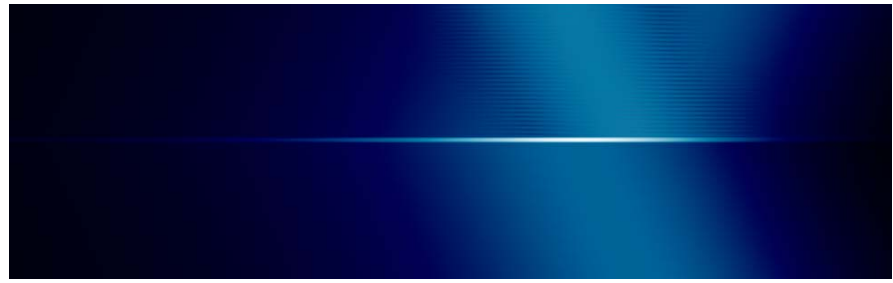

FIG. 7 Modulus of the field for a symmetrical slab with $\epsilon_{1}=\epsilon_{3}=9, \mu_{1}=\mu_{3}=1$, $\epsilon_{2}=-1.5, \mu_{2}=-1$. and $h=1.3 \lambda$ using a gaussian incident beam with a waist of $20 \lambda$ and an incidence angle of $\theta=22.78^{\circ}$. The pole corresponding to the leaky mode is located at $\alpha_{p}=(1.16823-0.01125 i) k_{0}$.

\section{THE GROUNDED LEFT-HANDED SLAB}

The grounded left-handed slab is a much more simple structure for (i) there is no need to distinguish two different types of leaky slab modes and (ii) the structure can not support any leaky surface mode. All the leaky modes are then slab modes and are found for $\alpha<n_{2} k_{0}$. The reflection coefficient of the grounded slab is given by Eq. (2) with $r_{23}=-1$ for the TE polarization and $r_{23}=1$ for the TM polarization so that the dispersion relation gives

$$
\left|r_{12}\right|=e^{2 \gamma_{2}^{\prime \prime} h} .
$$

Since $\left|r_{12}\right|>1$ then all the solutions of the dispersion relation are located in the lower part of the complex plane so that they are all backward.

It is then easy to show that the relation $r^{+}(z)^{*}=r\left(z^{*}\right)$ still holds. As a consequence, the leaky modes of a grounded left- handed slab and of its corresponding right-handed structure can be excited for the same angle of incidence of the impinging beam.

\section{CONCLUSION}

In this paper, we have thoroughly studied the leaky modes of a left-handed slab for realistic values of the permittivity and permeability of the left-handed medium $[7,19,20]$ which can be obtained using structures like split-ring resonators. Our results can be summarized as follows. Left-handed slab may support two types of leaky modes :

- Leaky slab modes, which are always backward because of the negative refraction phenomenon. When the transmission is not null, leaky modes of the left-handed slab and of its corresponding right-handed structure are excited for the same angle of incidence.

- Leaky surface modes, which may be backward or forward depending on the propagation direction of the surface wave itself.

This work could help to interpret many giant lateral shifts as excitations of exotic leaky waves $[12,13,16]$. Since the existence of backward slab waves is linked to the property of negative refraction, and since these leaky waves constitute a signature of a left-handed slab behaviour we think that they could be used to characterize the left-handedness of metamaterial or photonic crystal structures far better than other methods [21].

\section{ACKNOWLEDGEMENTS}

This work has been supported by the French National Agency for Research (ANR), project 030/POEM. The authors would like to thank Alexandru Cabuz and Kevin Vynck for their help.

\section{ANNEX}

In this annex, we will clearly define the choice we have made for the definition of the complex square root and prove that for $z$ on the first Riemann sheet (but not on the cut line) we have $\left|r_{i j}(z)\right|>1$ when media $i$ and $j$ are not both right-handed.

Since the square root can be continued on the complex plane, $r$ and $r_{i j}$ can be continued as well. We have chosen to take $\sqrt{z}=\sqrt{r} e^{i \frac{\theta}{2}}$ with $z=r e^{i \theta}$ and $\left.\left.\theta \in\right]-\pi, \pi\right]$, as a definition of the square root. This means that we have placed the cut line on the negative part of the real axis and if $x$ is a positive real, $\sqrt{-x}=i \sqrt{x}$. This defines the square root on the entire complex plane, to which we refer as the first Riemann sheet. When we write that $z$ is on the second Riemann sheet, it will mean that we have taken the opposite of $\sqrt{z}$ as defined above.

With this choice, we have (i) $\Re(\sqrt{z}) \geq 0$ (ii) $\sqrt{z^{*}}=\sqrt{z}^{*}$ for $z$ on both sheets but not on the cut line (iii) if $\Im(z)<0$, 
$\Im(\sqrt{z})<0$ and if $\Im(z)>0, \Im(\sqrt{z})>0$ (iv) the function $\gamma(z)=\sqrt{\epsilon \mu k_{0}^{2}-z^{2}}$ has a cut line on the real axis (on ]$\left.-\infty,-n k_{0}\right] \cup[n k 0,+\infty]$ more precisely) and the function $\gamma$ on the real axis is continuous with the part of the complex plane which is under the cut line: when $z$ passes through the cut line from the first Riemann sheet (coming from the lower part of the plane) to the second Riemann sheet, $\gamma(z)$ is continuous. When a function which can be written using $\gamma(z)$ presents a pole, it must be found either (i) for $z$ on the first Riemann sheet and under the real axis (we will say that the pole itself is on the first Riemann sheet in this case) or (ii) for $z$ on the second Riemann sheet but above the real axis.

We have

$$
r_{i j}=\frac{\kappa_{i}-\kappa_{j}}{\kappa_{i}+\kappa_{j}} .
$$

The modulus of $r_{i j}$ reads as

$$
\begin{aligned}
\left|r_{i j}\right|^{2} & =\frac{\left(\kappa_{i}-\kappa_{j}\right)\left(\kappa_{i}^{*}-\kappa_{j}^{*}\right)}{\left(\kappa_{i}+\kappa_{j}\right)\left(\kappa_{i}^{*}+\kappa_{j}^{*}\right)} \\
& =\frac{\left|\kappa_{i}\right|^{2}+\left|\kappa_{j}\right|^{2}-2\left(\kappa_{i}^{\prime} \kappa_{j}^{\prime}+\kappa_{i}^{\prime \prime} \kappa_{j}^{\prime \prime}\right)}{\left|\kappa_{i}\right|^{2}+\left|\kappa_{j}\right|^{2}+2\left(\kappa_{i}^{\prime} \kappa_{j}^{\prime}+\kappa_{i}^{\prime \prime} \kappa_{j}^{\prime \prime}\right)},
\end{aligned}
$$

where $\kappa=\kappa^{\prime}+i \kappa^{\prime \prime}$.

Let us define $x$ and $y$ the real and imaginary part of $z=x+i y$ on the first Riemann sheet. Let us assume that $x>0$. We have

$$
\gamma=\sqrt{n^{2} k_{0}^{2}-z^{2}}=\sqrt{n^{2} k_{0}^{2}-x^{2}+y^{2}-2 i x y} .
$$

If $y>0$, then $x y>0$ and thus $\Im\left(n^{2} k_{0}^{2}-z^{2}\right)<0$ so that finally $\Im(\gamma)<0$. If $y<0$, then $x y<0$ so that $\Im(\gamma)>0$. Since $\gamma(-z)=\gamma(z)$ the result will hold for $x<0$ too and for $x=0$, $\gamma(z)$ is real and positive so that the result obviously holds. So the imaginary part of $\gamma(z)$ is positive (resp. negative) when the imaginary part of $z$ is negative (resp. positive).

For any right-handed medium, $\kappa$ has the same property than $\gamma$. For a left-handed medium, since $\kappa=\frac{\gamma}{\mu}$ or $\kappa=\frac{\gamma}{\epsilon}$ depending on the polarization, the imaginary part of $\kappa$ has the sign of $\Im(z)$. Since $i$ and $j$ are not both right-handed, then $\kappa_{i}^{\prime \prime}$ and $\kappa_{j}^{\prime \prime}$ have not the same sign and the product $\kappa_{i}^{\prime \prime} \kappa_{j}^{\prime \prime}$ is always negative. Since $\Re(\sqrt{z})>0$ for all $z$ on the first Riemann sheet then $\kappa_{i}^{\prime} \kappa_{j}^{\prime}$ is always negative too.

Finally, since $\kappa_{i}^{\prime} \kappa_{j}^{\prime}+\kappa_{i}^{\prime \prime} \kappa_{j}^{\prime \prime}<0$, we have $\left|r_{i j}\right|>1$ for all $z$ except on the real axis. Please note that $r_{i j}$ is not, in the particular case of a left-handed medium, the reflection coefficient on the interface [22].

\section{References}

[1] V. Veselago, "The electrodynamics of substances with simultaneously negative values of $\epsilon$ and $\mu$ " Usp. Fiz. Nauk. 92, 517 (1967).

[2] R. Shelby, D. Smith, and S. Shultz, "Experimental verification of a negative index of refraction"Science 292, 77 (2001).
[3] I. Shadrivov, A. Sukhorukov, and Y. Kivshar, "Guided modes in negative-refractive-index waveguides" Phys. Rev. E 67, 057602 (2003).

[4] P. Tichit, A. Moreau, and G. Granet, "Localization of light in a lamellar structure with left-handed medium : the Light Wheel" Opt. Express 15, 14961-14966 (2007).

[5] J. Pendry, D. Schuring, and D. Smith, "Controlling Electromagnetic Fields" Science 312, 1780 (2006).

[6] T. Tamir, and H. Bertoni, "Lateral displacement of optical beams at multilayered and periodic structures" J. Opt. Soc. Am. 61, 1397 (1971).

[7] D. R. Smith, S. Schultz, P. Makos, and C. M. Soukoulis, "Determination of effective permittivity and permeability of metamaterials from reflection and transmission coefficients" Phys. Rev. B 65, 195104 (2002).

[8] F., and H. Haenchen, "Ein neuer und fundamentaler Versuch zur Totalreflexion" Ann. Phys. 1, 333 (1947).

[9] D. Felbacq, A. Moreau, and R. Smaali, "Coos-Haenchen effect in the gaps of photonic crystals" Opt. Lett. 28, 1633 (2003).

[10] A. Lakhtakia, "On plane wave remittances and Goos-Haenchen shifts of planar slabs with negative real permittivity and permeability" Electromagnetics 23, 71 (2003).

[11] P. Berman, "Coos-Haenchen shift in negatively refractive media" Phys. Rev. E 66, 067603 (2002).

[12] I. Shadrivov, A. A. Zharov, and Y. Kivshar, "Giant Goos-Haenchen effect at the reflection from left-handed materials" Appl. Phys. Lett. 83, 2713 (2002).

[13] L. Wang, and S. Zhu, "Large negative lateral shifts from the Kretschman-Raether configuration with left-handed materials" Appl. Phys. Lett. 87, 221102 (2005).

[14] A. Moreau, and D. Felbacq, “Comment on 'Large negative lateral shifts from the Kretschman-Raether configuration with left-handed materials'" Appl. Phys. Lett. 90, 066102 (2007).

[15] F. Pillon, H. Gilles, S. Girard, M. Laroche, R. Kaiser, and A. Gazibegovic, "Goos-Haenchen and Imbert-Fedorov shifts for leaky guided modes" J. Opt. Soc. Am. B 22, 1290 (2005).

[16] X. Chen, and C. Li, "Lateral shift of the transmitted light beam through a left-handed slab" Phys. Rev. E 69, 066617 (2004).

[17] R. Ruppin, "Surface polaritons of a left-handed medium" Phys. Lett. A 277, 61 (2000).

[18] I. Shadrivov, A. Sukhorukov, Y. Kishvar, A. Zharov, A. Boardman, and P. Egan, "Non-linear surface waves in left-handed materials" Phys. Rev. E 69, 016617 (2004).

[19] C. Soukoulis, T. Koschny, J. Zhou, M. Kafesak, and E. Economou, "Magnetic response of split ring resonators at terahertz frequencies" Phys. Stat. Sol. B 244, 1181-1187 (2007).

[20] S. 0'Brien, and J. B. Pendry, "Photonic band-gap effect and magnetic activity in dielectric composites" J. Phys. - Condens. Matter 14, 4035-4044 (2002).

[21] J. Kong, B. Wu, and Y. Zhang, "Lateral displacement of a Gaussian beam reflected from a grounded slab with negative permittivity and permeability" Appl. Phys. Lett. 80, 2084 (2002).

[22] D. Felbacq, and A. Moreau, "Direct evidence of negative refraction at media with negative $\varepsilon$ and $\mu^{\prime \prime}$ J. Opt. A : Pure Appl. 0p. 5, L9 (2003). 